\title{
Die sieben Kerneinflussfaktoren auf die Weiterentwicklung von Produktionsanlagen im Schrottrecycling
}

\author{
Torben Kraffczyk ${ }^{1}$ und Roland Pomberger ${ }^{2}$
}

${ }^{1}$ Geschäftsführung, REMONDIS Electrorecycling GmbH, Lünen, Deutschland

${ }^{2}$ Abfallverwertungstechnik und Abfallwirtschaft, Montanuniversität Leoben, Leoben, Österreich

Eingegangen 19. Januar 2021; angenommen 14. Februar 2021; online publiziert 16. März 2021

Zusammenfassung: Die vorliegende Systemkonzeptionierung von Metall- und Schrottrecyclinganlagen unter dem Einfluss umweltschutzrechtlicher Änderungen untersucht zunächst formalrechtliche, verfahrenstechnische sowie wirtschaftswissenschaftliche Aspekte des wertstromorientierten Schredderprozesses. Sie ist ein systemtheoretischer Ansatz, um die wesentlichen Einflussfaktoren auf die Weiterentwicklung von Schredderanlagen im Metall- und Schrottrecycling in einem Kreislaufmodell darzustellen.

Die Grundstruktur des Kreislaufmodells lässt sich weiter auf die Konzipierung branchenbegleitender Recyclinganlagen übertragen. Im Kontext der Corona-Pandemie werden zusätzliche Einflussfaktoren auf die Weiterentwicklung von Produktionsanlagen im Elektro- und Elektronikschrottrecycling diskutiert.

Der Fokus liegt dabei in der Analyse und Gewichtung technischer und sozialer Herausforderungen in Folge der Anwendung des europäischen und nationalen Regelwerks in Zeiten volatiler Marktstrukturen, im konjunkturellen Abwärtstrend der industriellen Wirtschaft und unter einem anwachsenden Wertstoffverfall des Inputstroms an diesen Abfallbehandlungsanlagen.

Schlüsselwörter: Schredder, Schrottrecycling, Elektroschrott, Sammelgruppen

System Concept of Production Design and Development in Scrap and Metal Recycling

Abstract: The present system concept of metal and scrap recycling plants deals with possible effects of renewed leg-

$\overline{\text { Dr.-Ing. T. Kraffczyk ( } \bowtie)}$

Geschäftsführung,

REMONDIS Electrorecycling $\mathrm{GmbH}$

Brunnenstr. 138,

44536 Lünen, Deutschland

torben.kraffczyk@remondis.de islative amendments and evaluates legal and technological as well as economical aspects of the value stream oriented shredder process. It is a system oriented approach to point out the main influencing factors on the development of shredder plants in scrap and metal recycling. Those factors are being merged into a superordinate system concept used to support strategic management decisions regarding future investments in the waste treatment sector. Under the given legal circumstances, the shredder process itself has to be resource-friendly, energy- and cost-efficient while still following the stated emission limit values.

Therefore, the conceptual focus lies in the argumentation and evaluation of technical challenges as a major result of the implementation of national and international legal terms in the age of volatile market conditions, present recession, and value decline in prematerial compositions. The system concept is the result of a research project from October 2016 to December 2019 at the TSR Recycling GmbH \& Co. KG, Lünen, Germany.

The concept itself forms a base for the development of plant technology in other metal recycling fields, such as e-waste recycling. Due to the current COVID-19 pandemic situation, the model is extended to other and further influencing factors and helps to understand the short lifespan of theoretical modelling within the field of scrap metal recycling

Keywords: Scrap metal recycling, Shredder, E-waste, Modelling

\section{Einleitung}

Mit einer Versorgungsquote von 20 bis $25 \%$ gehören Schredderanlagen zu den wesentlichen Elektrostahlwerkslieferanten und bilden einen Kernbereich der deutschen Recyclingwirtschaft für Eisen- und Metallschrotte. Europäische Normen und Rechtsvorschriften prägen die Kreislaufwirtschaft und den daran gebundenen Umweltschutz in 
Abb. 1: Die sieben Kerneinflussfaktoren auf die Weiterentwicklung von Schredderanlagen unter dem Einfluss umweltschutzrechtlicher Änderungen [2]

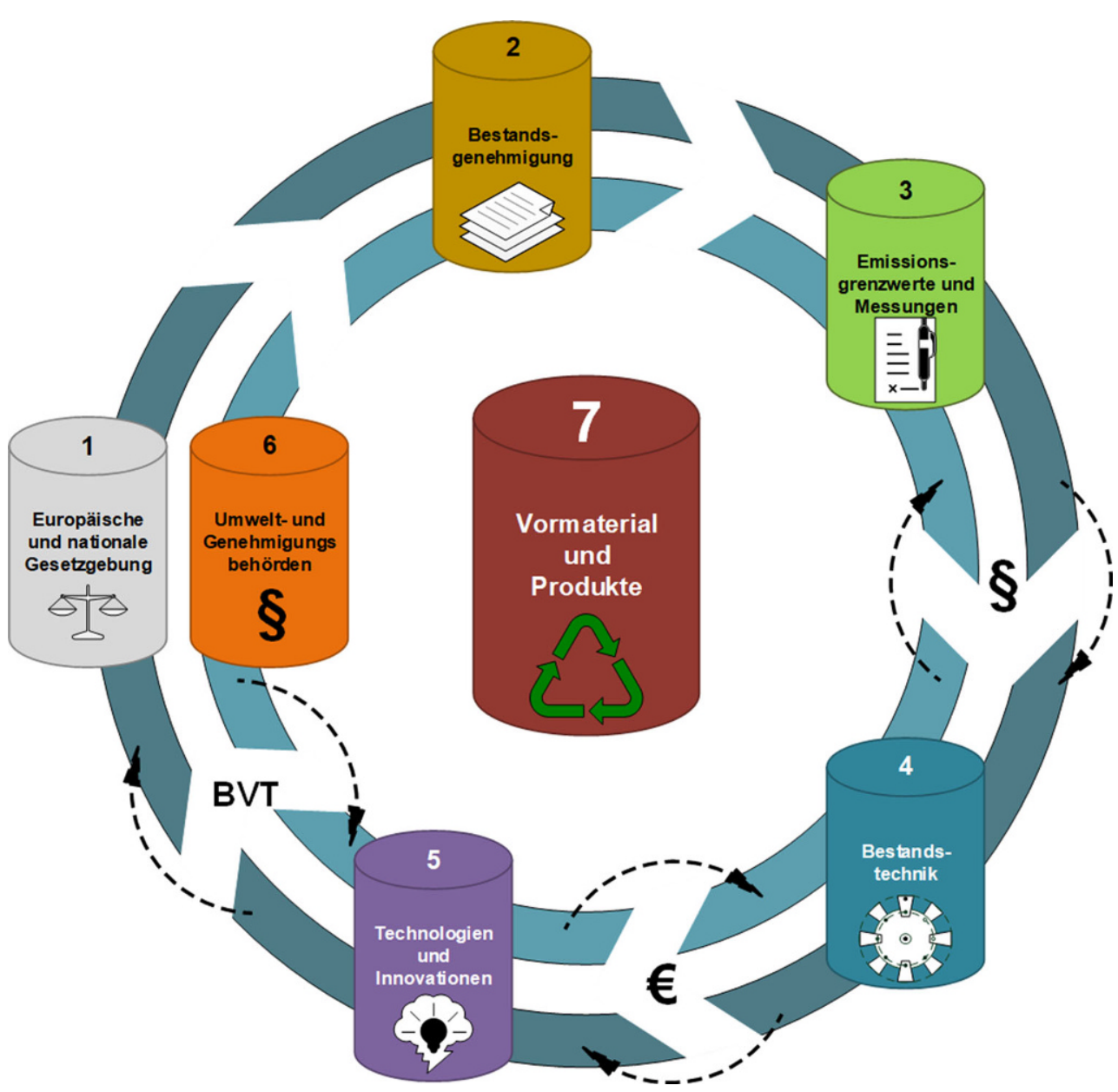

Deutschland, zunehmend auch in den Bereichen der Energieeffizienz, Ressourcenschonung und dem Klimaschutz [1] und nehmen nachhaltigen Einfluss auf den Fortbestand altgedienter Schredderanlagen. Darüber hinaus setzen Überkapazitäten im Markt, die Konjunkturschwäche der europäischen Wirtschaft und die wertstoffhaltigen Veränderungen im Vormaterial Schredderbetreiber in ihrer langfristigen strategischen Ausrichtung unter Druck.

Die methodische Risikobewertung von Langzeitinvestitionen im Altanlagensegment wird neben der betriebs- und umweltschutzrechtlichen Evaluation im Entwicklungsprozess von Schredderanlagen zunehmend systemrelevant. Die im Folgenden dargestellten sieben Kerneinflussfaktoren auf die Weiterentwicklung von Schredderanlagen sind ein Modellansatz, strategische Managemententscheidungen im Investitionsprozess zu lenken, und das übergeordnete Ergebnis einer dreijährigen Forschungsarbeit bei der TSR Recycling GmbH \& Co. KG in Deutschland im Zeitraum Oktober 2016 bis Dezember 2019 [2].

\section{Die sieben Kerneinflussfaktoren}

Die sieben Kerneinflussfaktoren auf die Weiterentwicklung von Schredderanlagen unter dem Einfluss umweltschutzrechtlicher Änderungen fußen auf den formalrechtlichen
Anpassungen des BREF (BVT-Merkblatt) für Abfallbehandlungsanlagen aus dem Jahre 2018 [3] und der novellierten Entwurfsfassung der TA Luft aus derselben Entwicklungsperiode [4]. Das in Abb. 1 dargestellte Kreislaufmodell stellt die Verbindung zwischen den gesetzlichen Herausforderungen und den strategischen Managemententscheidungen der Betreiber im Kontext einer Anlagenneuplanung dar [5]. Ein finaler Referentenentwurf der TA LuftNovelle aus dem Jahre 2020 soll nun letztlich auf nationaler Ebene verabschiedet werden, wobei die Änderungen zu der Fassung aus 2018 für Schredderbetreiber nur unwesentlich, aber überwiegend positiv sind.

Das Modell verfolgt die Theorie, dass die überarbeiteten Emissionsgrenzwertbestimmungen und Umweltauflagen des BREF und der TA Luft direkten oder indirekten Einfluss auf die technischen und betrieblichen Bestandsprozesse von Schredderanlagen nehmen.

Die in Säule 1 dargestellten rechtlichen europäischen und nationalen Rahmenbedingungen wirken sich bei Einflussnahme auf die Nebenbestimmungen des Genehmigungsbescheides (Säule 2) direkt auf den Fortbestand entsprechender IED-Anlagen aus. Genehmigungsrelevante emissionstechnische Betriebsgrenzen sind mit den neuen rechtlichen Emissionsgrenzwerten abzugleichen und indizieren infolgedessen, ob ein Eingriff in die Bestandstechnik 
in Form einer verfahrenstechnischen oder betrieblichen Maßnahme erforderlich ist.

Die kontinuierliche und/oder diskontinuierliche Überwachung sowie Nachweisführung der festgelegten Emissionsschwellen gegenüber den Behördenvertretern und Kontrollinstanzen bilden den dritten Kerneinflussfaktor. Unter der Prämisse verschärfter Umweltanforderungen wird ein Eingriff in die Bestandstechnik (Säule 4) genau dann erforderlich (u. U. in Form einer wesentlichen Ände-

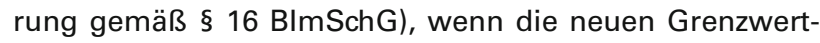
bestimmungen aus der Säule 1 im Altbestand nicht mehr gehalten werden können. Aus den technologischen Defiziten in der Bestandstechnik heraus ergibt sich schließlich die Frage nach einer Neuinvestition und die hiermit verbundenen planerischen Herausforderungen und ökonomischen Risiken, welche hier als systemischer Entwicklungsschritt von Säule 4 zu 5 dargestellt sind.

Die Weiterentwicklung produktionslastiger Industrieanlagen in der Abfallwirtschaft bietet im Kontext eines emissionstechnischen Fortentwicklungsgedankens nur wenige monetäre Anreize und ist eher gesetzlich bzw. behördengetrieben. Technologische Innovationen im Rahmen eines Optimierungsprozesses zu etablieren, wird dann reizvoll, wenn diese in Zusammenarbeit mit den Umwelt- und Genehmigungsbehörden (Säule 6) einen neuen besten verfügbaren Stand der Technik (BVT) festlegen.

Massenleistungsfähige Großschredderanlagen sind aus der Betrachtung eines klassisch linearen Wertstrommodells (Input-Prozess-Output) heraus dazu ausgelegt, hochkomplexe Stoffströme bei möglichst hoher Ausbringung zu verarbeiten. Die wesentliche Herausforderung in der Weiterentwicklung von Schredderanlagen besteht neben den formalrechtlichen Vorgaben besonders im wertstofflichen Verfall des Vormaterials bei steigenden Qualitätsanforderungen der Abnehmer an das Produkt (siebter Kerneinflussfaktor). Damit birgt die Weiterentwicklung von Schredder- anlagen zur Eisen-, Konsumgüter und Metallschrottaufbereitung vor allem wirtschaftliche Risiken.

Die Untersuchung der einzelnen Einflussfaktoren im Kontext des beschriebenen Modells ist dabei ein möglicher Weg, das unternehmerische Planungsrisiko kalkulatorisch abzubilden und die Managemententscheidung im Rahmen einer Neuinvestition zu erleichtern. Durch die Einzelgewichtung der verschiedenen Einflussfaktoren wird dem Schredderbetreiber ermöglicht, den für seinen Wirkbereich relevanten Kernaufwand zu bewerten und entsprechend notwendige Ressourcen gezielt einzusetzen [2].

\section{Adaption des Systemkonzepts im Elektroschrott}

Der systemische Modellansatz birgt darüber hinaus die Möglichkeit der Übertragung auf andere Geschäftsfelder in der Abfallwirtschaft, bedarf im Einzelfall jedoch einer Anpassung oder Erweiterung. Legt man die sieben Säulen beispielsweise auf einen Weiterentwicklungsprozess und eine damit verbundene Investitionsentscheidung im Kühlschrankrecycling (Wärmeüberträger), fallen neben den genannten formalrechtlichen äußeren Einflussfaktoren zusätzliche herstellergebundene Auditierungspflichten sowie Marktstrukturen und technologische Herausforderungen ins Gewicht, die im klassischen Schredderbetrieb keine oder eine geringfügigere Bedeutung finden. Abb. 2 fasst die wesentlichen Anpassungen im Systemkonzept bei der Weiterentwicklung von Produktionsanlagen der Sammelgruppe 1 (SG 1) im Elektronikschrott zusammen.

IED-Anlagen zur Aufbereitung von Elektro- und Elektronikschrotten sind auf nationaler Ebene besonders an das ElektroG gebunden, welches sich derzeit ebenfalls in einer Überarbeitungsphase befindet [6]. Ergänzt wird diese formalrechtliche Komponente durch fremd- oder eigensauferlegte Auditierungspflichten. Die Zuordnung der Auditie-
Abb. 2: DieAdaption von Kerneinflussfaktoren für das Elektronikschrottrecycling

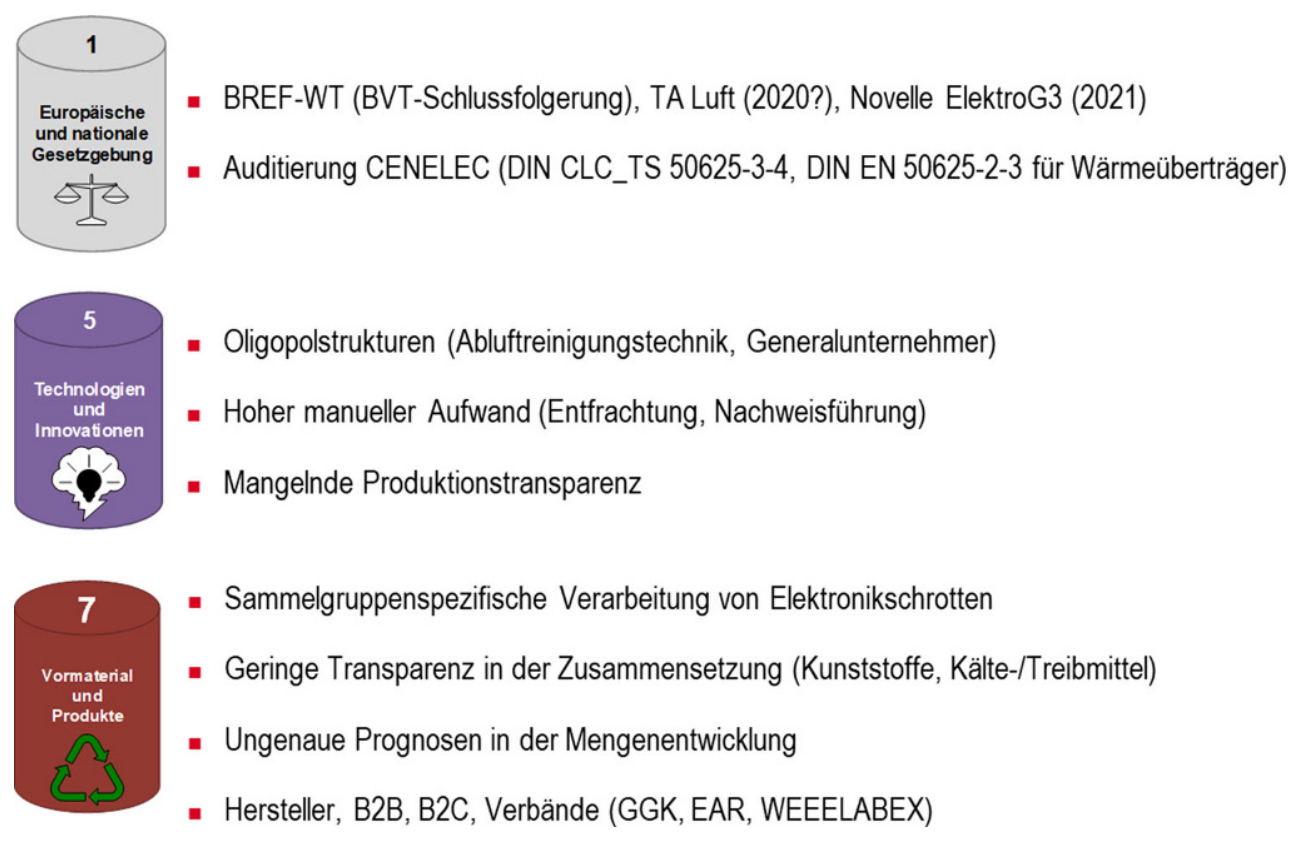


rung als Einflussgröße im Kreislaufmodell kann dabei als segregierter Faktor oder im Rahmen von Säule 1 erfolgen.

Gemessen an der messtechnischen Kontrollinstanz des Einflussfaktors 3 (siehe Abb. 1) können herstellergebundene Auflagen, die WEEELABEX-Zertifizierung oder der Betrieb einer Produktionslinie gemäß CENELEC-Standard [7, 8] sowohl Einzug in den dritten als auch fünften Kerneinflussfaktor finden und müssen im Kontext einer Investitionsentscheidung entsprechend gewichtet werden.

Die technische Weiterentwicklung von Bestandsystemen und/oder deren Neubau erfolgt i.d.R. unter einer Beteiligung weniger Systemlieferanten. Die geminderte Angebotsstruktur nimmt Einfluss sowohl auf die Investitionskosten als auch die Entwicklungsgeschwindigkeit entsprechender Nischenprodukte. Eine Rückkopplung gesetzlicher
Vorgaben in die technische Umsetzung erfolgt primär anwendergetrieben.

Die Behandlung der SG 1 ist bereits im Rahmen der Vorentfrachtung stark durch manuelle Arbeitsabläufe geprägt (u.a. strichlistengebundene Nachweisführung der Entsorgungsmengen). Aber auch das Handling von Kleingeräten der SG 5 erfordert zunehmend manuelle Vorsortierungsund Entfrachtungsschritte $u$. a. auf Grund der steigenden Inputmengen an verbauten Li-lon-Akkus. Die Weiterentwicklung von technischen Rückbauzentren (RBZ) verlangt folglich eine gesunde Kombination aus automatisierten und manuellen Abläufen. Dabei soll das mittelfristige Ziel sein, die händische Datenerfassung auf ein digitales Level zu heben.
Abb. 3: Beispielhafte Auswertung der Input- und Produktionsmengen im Jahr 2020 unter Berücksichtigung der Produktivität
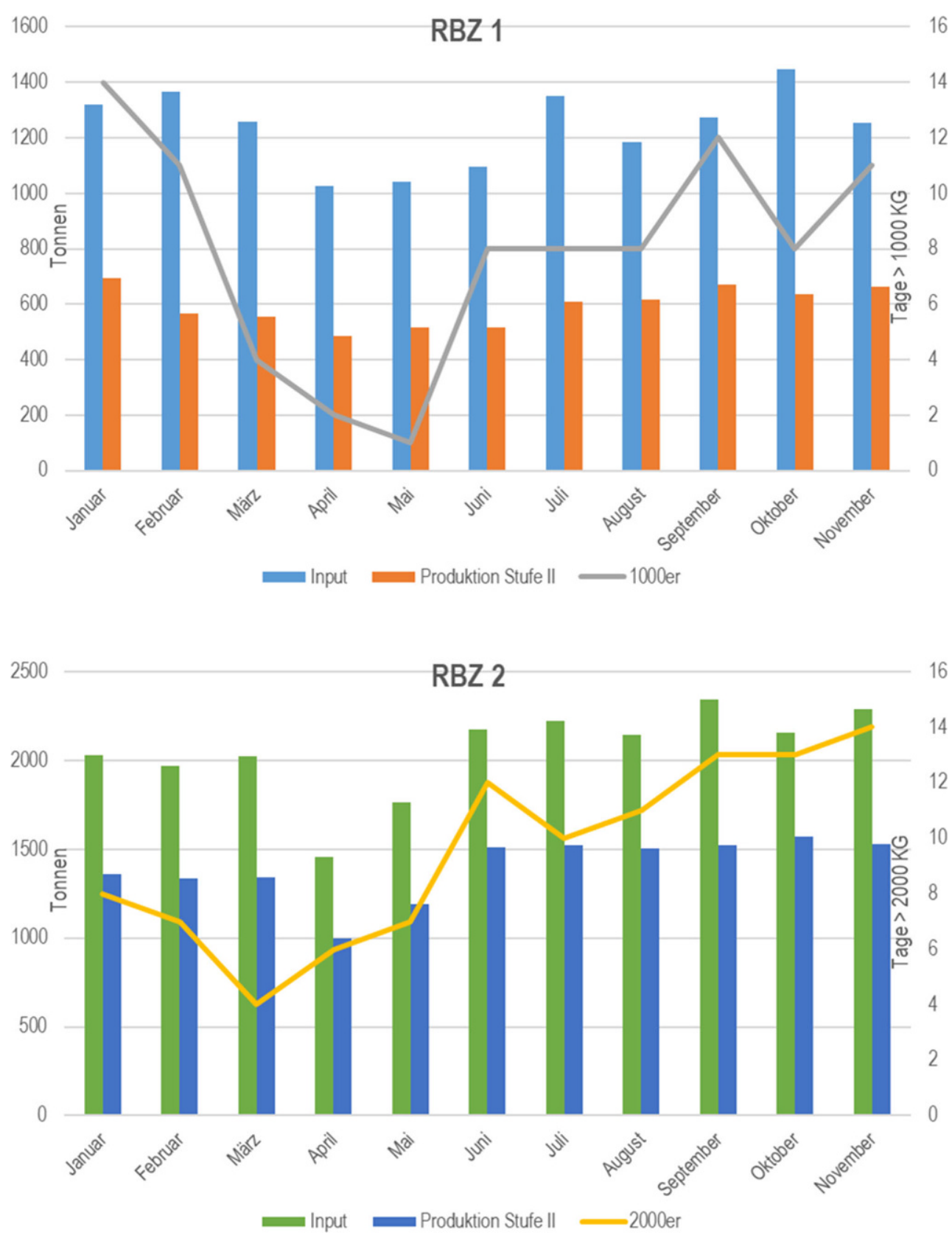
Die Forderung nach einer sammelgruppenspezifischen verfahrenstechnischen Behandlung von Elektronikschrotten führt zu einer monetären Doppelbelastung bei sammelgruppenweiten Weiterentwicklungsstrategien. Es ist möglich, dass sich Marktbegleiter zukünftig auf eine SG spezialisieren werden, um den Stand der BVT langfristig in den einzelnen RBZ gerecht zu werden.

Die Mengenentwicklung in der SG 1 und 5 war besonders im Jahr 2020 unter den Einflüssen der Corona-Pandemie nicht nachhaltig prognostizierbar. Wo Produktdesign und Produktentwicklung ohnehin zunehmend komplexer werden - speziell im Einsatz von Kunststoffen oder Kältemittekompositionen (Frigene) in Wärmeüberträgern - haben die häuslichen Aufräumarbeiten während der Heimarbeit sowie überdurchschnittlich hohe Ausgaben (eingespartes Urlaubsgeld) in Kühl-, Gefrierschränke und Konsumgüter dazu geführt, dass längst vergessen geglaubte Altprodukte wieder in die Abfallwirtschaft Einzug erhalten haben.

Neben der Komplexität der kommunalen und direkten Vertriebswege bleibt durch die Einflüsse der weltweiten Pandemie auch zu Beginn des Jahres 2021 weiterhin ungewiss, wie lange sich das hohe Mengenaufkommen von Elektroschrotten schlussendlich halten wird.
Auch im Kontext der geforderten Rücknahmepflichten von Altgeräten in Supermärkten bleibt abzuwarten [9], wie schnell und um welchen Wert ein Rückgang des aktuellen Niveaus (speziell der SG 5) in Deutschland zu verzeichnen sein wird.

\section{Säule 8 als neue Erkenntnis im Kreislauf- modell}

Die globalen Ereignisse des Jahres 2020 haben eine neue Einflussgröße in das bestehende Systemkonzept zur Weiterentwicklung von Produktionsanlagen im Schrottrecycling gebracht. Äußere Einflüsse in Form der höheren Gewalt haben eine neue Bedeutung für die Risikoevaluation bei Großinvestitionen in der gegenwärtigen Branche eingenommen.

Die Schließung von Wertstoffhöfen im französischen Ausland hat besonders in den Monaten April und Mai 2020 zu einem nahezu kompletten Rückgang der Eingangsmengen in den RBZ geführt. Die anfänglichen Mengeneinbußen in Deutschland haben sich über die einzelnen SG verteilt, ab Juni wieder erholt, wie in Abb. 3 (RBZ 1 und 2) verdeutlicht.

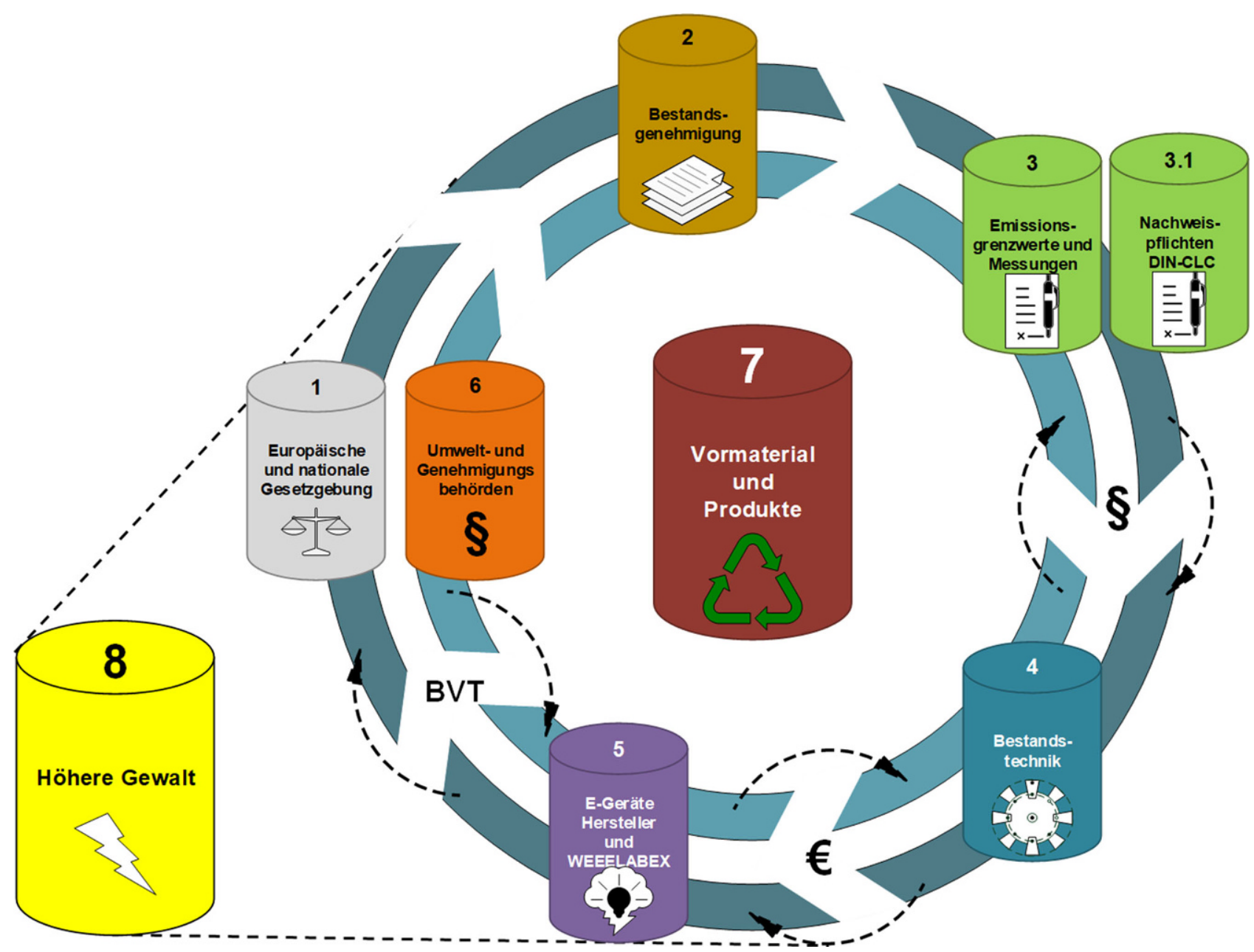

Abb. 4: Die acht Kerneinflussfaktoren auf die Weiterentwicklung von Elektronikschrottrecyclinganlagen im Jahre 2020 
Diese Entwicklungen waren zu diesem Zeitpunkt weder planbar noch im aktuellen Systemkonzept berücksichtigt.

Die Grafiken in Abb. 3 zeigen darüber hinaus, dass trotz positivem Materialinput und Personalbestand eine degressive Leistungsentwicklung pro Mitarbeiter in den Monaten März bis Mai 2020 zu verzeichnen ist. Deutlich wird dieser Produktivitätsverlust in der SG 1-Line durch die fallende Anzahl an Tagen, an denen weniger als die geforderte Benchmarkmenge (1000er- und 2000er-Linie) an Altkühlgeräten in der Verarbeitungsstufe II erreicht wurde.

Diese "Corona-Depression" ist ein operativer Störfaktor, der im Rahmen einer anlagenspezifischen Planungsprämisse zur Verunsicherung führen kann.

Die obigen Fakten gegeben, lässt sich das Systemkonzept zur Weiterentwicklung von Produktionsanlagen im Schrottrecycling um einen weiteren wesentlichen Kerneinflussfaktor ergänzen, dessen Gewichtung ebenfalls individuell als Einzelgröße zu gestalten ist und im nachstehenden Kreislaufmodell eine übergeordnete Rolle einnimmt - "Höhere Gewalt".

Abb. 4 stellt die acht Kerneinflussfaktoren auf die Weiterentwicklung von Elektro- und Elektronikschrottrecyclinganlagen beispielhaft systematisch dar.

\section{Schlussfolgerung}

Das Recycling an Schredderanlagen ist ein reaktives Wertschöpfungsmodell und dafür ausgelegt, abnehmergelenkt sehr komplexe Schrotte aufzubereiten. Dieser Aufbereitungsprozess ist zunehmend durch steigende Entsorgungskosten und Ausbringungsverluste sowie Absatzschwierigkeiten geprägt und stellt ein erhöhtes unternehmerisches Risiko im europäischen Wettbewerbsvergleich dar.

Systemkonzeptionierungen in Form des beschriebenen Kreislaufmodells sind ein hilfreiches strategisches Mittel, Weiterentwicklungen an Produktionsanlagen des Metallund Schrottrecyclings und damit verbundene Investitionsentscheidungen im Rahmen eines sich stetig wandelnden Umwelt- und Marktgeschehens zu bewerten.

Die Erkenntnisse aus der Pandemie des Jahres 2020/2021 verdeutlichen des Weiteren, dass systemtheoretische Modelle immer nur so gut sind wie die Zeit, in die sie hineinpassen.

Eine kontextbezogene Adaption von Basismodellen ist ohnehin dort unabdingbar, wo sich Rahmenparameter verändern. Weil es in der Praxis i.d.R. immer mindestens eine
Unbekannte gibt, die man in der Theorie vorher bereits erkennen hätte müssen, bietet es sich in einer Planungsphase an, mehrere Systemkonzepte unter Verwendung weiter spezifischer Kerneinflussfaktoren nebeneinander aufzubauen, um das Unvorhergesehene ggf. sichtbar zu machen.

\section{Literatur}

1. Birnstengel, B.; Eckhardt, M.; Häusler, A.; Hoffmeister, J.; Labinsky, A.; Lambert, J.: Statusbericht der deutschen Kreislaufwirtschaft: Einblicke und Aussichten. https://www.prognos.com/uploads/tx atwpubdb/Prognos_Statusbericht_2018.pdf (19.11.2019)

2. Kraffczyk, T.: Systemkonzeptionierung von Großschredderanlagen im Metall- und Schrottrecycling unter dem Einfluss umweltschutzrechtlicher Änderungen, Clausthal-Zellerfeld: Papierflieger Verlag $\mathrm{GmbH}, 2020$

3. Pinasseau, A.; Zerger, B.; Roth, J.; Canova, M.; Roudier, S.: Best Available Techniques (BAT) Reference Document for Waste Treatment Industrial Emissions Directive 2010/75/EU (Integrated Pollution Prevention and Control) EUR 29362 EN, Luxembourg: Publications Office of the European Union, 2018

4. Bundesministerium für Umwelt, Naturschutz und nukleare Sicherheit (BUNS): Neufassung der Ersten Allgemeinen Verwaltungsvor schrift zum Bundes-Immissionsschutzgesetz (Technische Anleitung zur Reinhaltung der Luft - TA Luft), Entwurfsfassung, 2018. https:// www.bmu.de/fileadmin/Daten BMU/Download_PDF/Glaeserne Gesetze/19._Lp/ta_luft/entwurf/ta_luft_180716_refe_bf.pdf. Zugegriffen: 15.01.2021

5. Kraffczyk, T.; Goldmann, D.: Der Einfluss von umweltrechtlichen Änderungen auf die Weiterentwicklung von Schredderanlagen, World of Metallurgy - ERZMETALL, 71 (2018), Nr. 4, S. 214-220

6. Hesselmann Service GmbH: Bundeskabinett beschließt Änderung des Elektrogesetzes. https://www.elektrogesetz.de/bundeskabinettbeschliesst-aenderung-des-elektrogesetzes/. Zugegriffen: 15.01.2021

7. Europäisches Komitee für Elektrotechnische Normung (EK): DIN CLC/TS 50625-2-3 (VDE V 0042-13-23:2018-05), Sammlung, Logistik und Behandlung von Elektro- und Elektronik-Altgeräten (WEEE) Teil 2-3: Anforderungen an die Behandlung von Wärmeüberträgern und anderen Elektro- und Elektronik-Altgeräten, die VFC und/oder VHC enthalten, 2017

8. Europäisches Komitee für Elektrotechnische Normung (EK): DIN CLC/TS 50625-3-4 (VDE V 0042-13-34:2018-07), Sammlung, Logistik und Behandlung von Elektro- und Elektronik-Altgeräten (WEEE) Teil 3-4: Spezifikation der Schadstoffentfrachtung - Wärmeüberträger, 2017

9. EUWID Europäischer Wirtschaftsdienst GmbH: ElektroG-Novelle führt Rücknahmepflicht für Altgeräte in Supermärkten ein. https://www.euwid-recycling.de/news/politik/einzelansicht/Artikel/ elektrog-novelle-fuehrt-ruecknahmepflicht-fuer-altgeraete-in-super maerkten-ein.html. Zugegriffen: 15.01.2021

Hinweis des Verlags. Der Verlag bleibt in Hinblick auf geografische Zuordnungen und Gebietsbezeichnungen in veröffentlichten Karten und Institutsadressen neutral. 